\title{
ARITHMETICAL STRUCTURES ON GRAPHS WITH CONNECTIVITY ONE
}

\author{
HUGO CORRALES AND CARLOS E. VALENCIA
}

\begin{abstract}
Given a graph $G$, an arithmetical structure on $G$ is a pair of positive integer vectors $(\mathbf{d}, \mathbf{r})$ such that $\operatorname{gcd}\left(\mathbf{r}_{v} \mid v \in V(G)\right)=1$ and

$$
(\operatorname{diag}(\mathbf{d})-A) \mathbf{r}=0
$$

where $A$ is the adjacency matrix of $G$. We describe the arithmetical structures on graph $G$ with a cut vertex $v$ in terms of the arithmetical structures on their blocks. More precisely, if $G_{1}, \ldots, G_{s}$ are the induced subgraphs of $G$ obtained from each of the connected components of $G-v$ by adding the vertex $v$ and their incident edges, then the arithmetical structures on $G$ are in one to one correspondence with the $v$-rational arithmetical structures on the $G_{i}$ 's. We introduce the concept of rational arithmetical structure, which corresponds to an arithmetical structure where some of the integrality conditions are relaxed.
\end{abstract}

\section{INTRODUCTION}

Given a multidigraph $G=(V, E)$ and an integral positive vector $\mathbf{d}$, let

$$
L(G, \mathbf{d})_{u, v}= \begin{cases}\mathbf{d}_{u} & \text { if } u=v, \\ -m_{u, v} & \text { if } u \neq v,\end{cases}
$$

where $m_{u, v}$ is the number of the directed arcs between $u$ and $v$. If $\mathbf{d}$ is the degree vector of $G$, then $L(G, \mathbf{d})$ is the Laplacian matrix of $G$, which is a matrix with rank $|V|-1$ and nullspace spanned by the all-ones vector 1. An arithmetical structure on $G$ is a pair $(\mathbf{d}, \mathbf{r}) \in \mathbb{N}_{+}^{|V|} \times \mathbb{N}_{+}^{|V|}$ such that

$$
L(G, \mathbf{d}) \mathbf{r}^{t}=\mathbf{0}^{t} \quad \text { and } \quad \operatorname{gcd}\left(\mathbf{r}_{v}\right)_{v \in V}=1 .
$$

Thus, the notion of arithmetical structures on $G$ generalizes the Laplacian matrix of $G$. The arithmetical structures on simple graphs were introduced by Lorenzini in [6] as some intersection matrices that arise in the study of degenerating curves in algebraic geometry. More precisely, the vertices of $G$ represent the components of a degeneration of a given curve, the edges represent the intersections of the components, and the entries of $\mathbf{d}$ are their self-intersection numbers. Lorenzini proved in $[6$ that if $G$ is a simple connected graph, then there are a finite number of arithmetical structures. In [4 this result was generalized to strongly connected multidigraphs. On the other hand, given an arithmetical structure $(\mathbf{d}, \mathbf{r})$ on $G$, let

$$
K(G, \mathbf{d}, \mathbf{r})=\operatorname{ker}\left(\mathbf{r}^{t}\right) / \operatorname{Im} L(G, \mathbf{d})^{t}
$$

be its critical group, which generalizes the concept of the critical group of $G$ introduced in [1]. Moreover, recently in [9] there was defined a sandpile group for the matrices $L(G, \mathbf{d})$ obtained from arithmetical structures. The Laplacian matrix of a graph is very important in spectral graph theory and in general in algebraic graph theory, see for instance [5] and the references therein.

2010 Mathematics Subject Classification. Primary 15B36; Secondary 14C17, 11C20, $11 \mathrm{D} 72$.

Key words and phrases. Arithmetical graphs, M-matrices, Laplacian matrix, Connectivity one, Cut vertex, 2-connected components.

The authors were partially supported by SNI. 
Since the number of arithmetical structures on any strongly connected multidigraph is finite, it seems possible to describe them. More precisely, given a connected graph $G$, we are interested in describing the set

$$
\mathcal{A}(G)=\left\{(\mathbf{d}, \mathbf{r}) \in \mathbb{N}_{+}^{|V|} \times \mathbb{N}_{+}^{|V|} \mid(\mathbf{d}, \mathbf{r}) \text { is an arithmetical structure on } G\right\} .
$$

In 2 there was described in detail the combinatorics of the arithmetical structures on the path and cycle graphs. Also, in [4] there was introduced and studied the arithmetical structures in the general setting of $M$-matrices and in particular the arithmetical structures on complete graphs, paths, and cycles were studied. Moreover, there was described a subset of the arithmetical structures on the cliquestar transformation of a graph $G$ in dependence on the arithmetical structures on $G$. Apart from the arithmetical structures on the path and cycle, in general the description of the arithmetical structures on a graph is a very difficult problem. For instance, for the complete and star graph, its arithmetical structures are in one to one correspondence with a variant of the Egyptian fractions. Therefore is important to have an idea of the complexity of the arithmetical structures on a graph with a cut vertex.

In this article we study the arithmetical structures on a connected graph with a cut vertex. The main results of this article are contained in Section 2, where we establish the relation between the arithmetical structures on a graph with one cut vertex and the arithmetical structures on their blocks. More precisely, given connected graphs $G_{1}$ and $G_{2}$ and a vertex $v_{i}$ of $G_{i}$, let $G_{1} \vee_{v} G_{2}$ be the graph that results if $v_{1}$ and $v_{2}$ are identified to a new vertex $v$. Moreover, let $\mathcal{A}_{v_{i}}\left(G_{i}\right)$ be the arithmetical structures on $G_{i}$ in which the integrality condition over $\mathbf{d}_{v_{i}}$ has been relaxed. Given $\left(\mathbf{a}, \mathbf{r}_{1}\right) \in \mathcal{A}_{v_{1}}\left(G_{1}\right)$ and $\left(\mathbf{b}, \mathbf{r}_{2}\right) \in \mathcal{A}_{v_{2}}\left(G_{2}\right)$, let

$$
l\left(\mathbf{r}_{1}, \mathbf{r}_{2}\right)= \begin{cases}\frac{l}{\left(\mathbf{r}_{1}\right)_{v_{1}}} \cdot\left(\mathbf{r}_{1}\right)_{u} & \text { if } u \in V\left(G_{1}\right) \\ \frac{l}{\left(\mathbf{r}_{2}\right)_{v_{2}}} \cdot\left(\mathbf{r}_{2}\right)_{u} & \text { if } u \in V\left(G_{2}-v_{2}\right),\end{cases}
$$

where $l=\operatorname{lcm}\left(\left(\mathbf{r}_{1}\right)_{v_{1}},\left(\mathbf{r}_{2}\right)_{v_{2}}\right)$. The main result of this article is the following description of the arithmetical structures on $G$ in terms of the arithmetical structures on $G_{1}$ and $G_{2}$.

Theorem 2.2. If $G_{1}$ and $G_{2}$ are connected graphs and $v_{i}$ is a vertex of $G_{i}$, then

$$
\mathcal{A}\left(G_{1} \vee_{v} G_{2}\right)=\left\{\left(\mathbf{a}+{ }_{v} \mathbf{b}, l\left(\mathbf{r}_{1}, \mathbf{r}_{2}\right)\right) \mid \mathbf{a}+{ }_{v} \mathbf{b} \in \mathbb{N}_{+}^{|V(G)|} \text { and }\left(\mathbf{a}, \mathbf{r}_{1}\right) \in \mathcal{A}_{v_{1}}\left(G_{1}\right),\left(\mathbf{b}, \mathbf{r}_{2}\right) \in \mathcal{A}_{v_{2}}\left(G_{2}\right)\right\},
$$

where

$$
\left(\mathbf{a}+{ }_{v} \mathbf{b}\right)_{u}= \begin{cases}\mathbf{a}_{u}+\mathbf{b}_{u} & \text { if } u=v \\ \mathbf{a}_{u} & \text { if } u \in V\left(G_{1}-v\right), \\ \mathbf{b}_{u} & \text { if } u \in V\left(G_{2}-v\right) .\end{cases}
$$

A typical case of a graph with cut vertices is a tree. In Section 3 we apply our result to the problem of describing the arithmetical structures on a tree. We give a description of the arithmetical structures on a star graph and give a partial description of the arithmetical structures on a general tree in terms of its star graphs.

In general we give the following description of the arithmetical structures on a tree. Given a tree $T$, let $\vec{T}$ be the digraph obtained from $T$ by replacing each edge $u v$ of $T$ by the arcs $u v$ and $v u$. Let $a: E(\vec{T}) \rightarrow \mathbb{Q}_{+}$ be a weight function on the arcs of $\vec{T}$.

Proposition 3.1. If $T$ is a tree, then

$$
\mathcal{A}(T)=\left\{(\mathbf{d}, \mathbf{r}) \in \mathbb{N}_{+}^{|V(T)|} \times \mathbb{N}_{+}^{|V(T)|} \mid \sum_{u v \in E(T)} \mathbf{a}_{u, v}=\mathbf{d}_{v} \forall v \in V(T) \text { and } \mathbf{a}_{u, v} \mathbf{a}_{v, u}=1 \forall u v \in E(T)\right\} .
$$


Finally, in the particular case that $T$ is a star graph, we get the following description.

Corollary 3.2. If $S_{m}$ is the star graph with center at $v$ and with $1, \ldots, m$ its leaves, then

$$
\mathcal{A}\left(S_{m}\right)=\left\{(\mathbf{d}, \mathbf{r}) \in \mathbb{N}_{+}^{m+1} \times \mathbb{N}_{+}^{m+1} \mid \mathbf{d}_{v}=\sum_{i=1}^{m} \frac{1}{\mathbf{d}_{i}}, \mathbf{r}_{v}=\operatorname{lcm}\left\{\mathbf{d}_{i}\right\}_{i=1}^{m} \text { and } \mathbf{r}_{i}=\frac{\mathbf{r}_{v}}{\mathbf{d}_{i}}\right\} .
$$

\section{Arithmetical structures on Graphs With CONneCtivity one}

Given two connected graphs $G_{1}, G_{2}$ and $v_{i}$ a vertex of $G_{i}$, let

$$
G=G_{1} \vee_{v} G_{2}
$$

be the graph obtained from $G_{1}$ and $G_{2}$ by identifying the vertices $v_{1}$ and $v_{2}$ to a new vertex, denoted by $v$. That is, $v$ is a cut vertex of $G$.

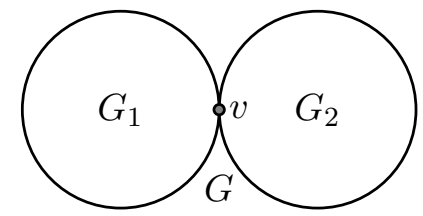

Figure 1. A graph with a cut vertex.

Lemma 2.1. Let $G_{1}$ and $G_{2}$ be two connected graphs and $v_{i}$ a vertex of $G_{i}$. If $G$ is equal to $G_{1} \vee_{v} G_{2}$, then

$$
\left.\operatorname{det}(G, X)\right|_{x_{v}=x_{v_{1}}+x_{v_{2}}}=\operatorname{det}\left(G_{1}-v_{1}, X\right) \cdot \operatorname{det}\left(G_{2}, X\right)+\operatorname{det}\left(G_{2}-v_{2}, X\right) \cdot \operatorname{det}\left(G_{1}, X\right),
$$

where $\operatorname{det}(G, X)$ is the determinant of the generalized Laplacian matrix

$$
L(G, X)_{u, v}= \begin{cases}x_{u} & \text { if } u=v \\ -m_{u, v} & \text { if } u \neq v\end{cases}
$$

with $x_{u}$ an indeterminate indexed by the vertex $u$.

Proof. Let $D$ be the digraph obtained from $G$ by replacing each edge $u v$ of $G$ by the arcs $u v$ and $v u$. Also, given $U \subseteq V(D)$, let $D[U]$ be the induced subgraph of $D$ by $U$ and let $D[U]^{w l}$ be the graph obtained from $D[U]$ when we delete any possible loops.

By [3. Theorem 4.1] we have that

$$
\begin{aligned}
\operatorname{det}(L(G, X)) & =\operatorname{det}(L(D, X))=\sum_{U \subseteq V(D)} \operatorname{det}\left(-A\left(D[U]^{w l}\right)\right) \prod_{u \notin U} x_{u} \\
& =\sum_{v \notin U \subseteq V(D)} \operatorname{det}\left(-A\left(D[U]^{w l}\right)\right) \prod_{u \notin U} x_{u}+\sum_{v \in U \subseteq V(D)} \operatorname{det}\left(-A\left(D[U]^{w l}\right)\right) \prod_{u \notin U} x_{u} \\
& =x_{v} \sum_{U \subseteq V(D-v)} \operatorname{det}\left(-A\left(D[U]^{w l}\right)\right) \prod_{v \neq u \notin U} x_{u}+\sum_{v \in U \subseteq V(D)} \operatorname{det}\left(-A\left(D[U]^{w l}\right)\right) \prod_{u \notin U} x_{u} .
\end{aligned}
$$

Now, let $\overrightarrow{\mathcal{F}}$ be the set of spanning directed 1-factors of $D$ (a directed 1-factor of $D$ is a subdigraph $C$ such that $d_{C}^{+}(v)=d_{C}^{-}(v)=1$ for all $\left.v \in V(C)\right)$ and let $c(C)$ be the number of connected components of $C$. 
Since $\operatorname{det}(-A(D))=\sum_{C \in \overrightarrow{\mathcal{F}}}(-1)^{c(C)}$ and the connected directed 1-factors of $D$ can not contain vertices of $G_{1}-v_{1}$ and $G_{2}-v_{2}$ (because $v$ is a cut vertex of $D$ ), then

$$
\begin{aligned}
\left.\operatorname{det}(L(G, X))\right|_{x_{v}=x_{v_{1}}+x_{v_{2}}} & =\left(x_{v_{1}}+x_{v_{2}}\right) \operatorname{det}\left(\mathrm{L}\left(\mathrm{G}_{1}-\mathrm{v}_{1}, \mathrm{X}\right)\right) \operatorname{det}\left(\mathrm{L}\left(\mathrm{G}_{2}-\mathrm{v}_{2}, \mathrm{X}\right)\right) \\
& +\operatorname{det}\left(\mathrm{L}\left(\mathrm{G}_{1}-\mathrm{v}_{1}, \mathrm{X}\right)\right)\left[\sum_{v \in U \subseteq V\left(G_{2}\right)} \operatorname{det}\left(-A\left(D[U]^{w l}\right)\right) \prod_{u \notin U} x_{u}\right] \\
& +\operatorname{det}\left(\mathrm{L}\left(\mathrm{G}_{2}-\mathrm{v}_{2}, \mathrm{X}\right)\right)\left[\sum_{v \in U \subseteq V\left(G_{1}\right)} \operatorname{det}\left(-A\left(D[U]^{w l}\right)\right) \prod_{u \notin U} x_{u}\right] \\
& =\operatorname{det}\left(\mathrm{L}\left(\mathrm{G}_{1}-\mathrm{v}_{1}, \mathrm{X}\right)\right)\left[x_{v_{2}} \operatorname{det}\left(\mathrm{L}\left(\mathrm{G}_{2}-\mathrm{v}_{2}, \mathrm{X}\right)\right)+\sum_{v \in U \subseteq V\left(G_{2}\right)} \operatorname{det}\left(-A\left(D[U]^{w l}\right)\right) \prod_{u \notin U} x_{u}\right] \\
& +\operatorname{det}\left(\mathrm{L}\left(\mathrm{G}_{1}-\mathrm{v}_{1}, \mathrm{X}\right)\right)\left[x_{v_{1}} \operatorname{det}\left(\mathrm{L}\left(\mathrm{G}_{1}-\mathrm{v}_{1}, \mathrm{X}\right)\right)+\sum_{v \in U \subseteq V\left(G_{1}\right)} \operatorname{det}\left(-A\left(D[U]^{w l}\right)\right) \prod_{u \notin U} x_{u}\right] \\
& =\operatorname{det}\left(G_{1}-v_{1}, X\right) \operatorname{det}\left(G_{2}, X\right)+\operatorname{det}\left(G_{2}-v_{2}, X\right) \operatorname{det}\left(G_{1}, X\right) .
\end{aligned}
$$

Before our principal result is presented, we need to introduce some notation. Given $\mathbf{a} \in \mathbb{R}^{V\left(G_{1}\right)}$ and $\mathbf{b} \in \mathbb{R}^{V\left(G_{2}\right)}$, let

$$
\left(\mathbf{a}+{ }_{v} \mathbf{b}\right)_{u}= \begin{cases}\mathbf{a}_{u}+\mathbf{b}_{u} & \text { if } u=v, \\ \mathbf{a}_{u} & \text { if } u \in V\left(G_{1}-v\right), \\ \mathbf{b}_{u} & \text { if } u \in V\left(G_{2}-v\right) .\end{cases}
$$

Moreover, given $A_{1} \subset \mathbb{R}^{V\left(G_{1}\right)}$ and $A_{2} \subset \mathbb{R}^{V\left(G_{2}\right)}$, let

$$
A_{1}+{ }_{v} A_{2}=\left\{\mathbf{a}+{ }_{v} \mathbf{b} \mid \mathbf{a} \in A_{1} \text { and } \mathbf{b} \in A_{2}\right\} .
$$

Now, given a set $U$ of vertices of $G$, we introduce the concept of a $U$-rational arithmetical structure.

Definition 2.2. A pair $(\mathbf{d}, \mathbf{r}) \in \mathbb{Q}_{+}^{n} \times \mathbb{N}_{+}^{n}$ is a $U$-rational arithmetical structure on $G$ whenever

$$
L(G, \mathbf{d}) \mathbf{r}^{t}=\mathbf{0}^{t}
$$

and $\mathbf{d}_{w} \in \mathbb{N}_{+}$for all $w \notin U$.

Let $\mathcal{A}_{U}(G)$ be the set of $U$-rational arithmetical structures on $G$. That is, $\mathcal{A}_{U}(G)$ is the set of arithmetical structures on $G$ where the integrality condition over the vectors of $U$ is relaxed. Clearly $\mathcal{A}(G) \subseteq \mathcal{A}_{U}(G)$. Sometimes we will, for simplicity, only refer to them as rational arithmetical structures if the set of vertices of $U$ is clear. Given $\left(\mathbf{a}, \mathbf{r}_{1}\right) \in \mathcal{A}_{v_{1}}\left(G_{1}\right)$ and $\left(\mathbf{b}, \mathbf{r}_{2}\right) \in \mathcal{A}_{v_{2}}\left(G_{2}\right)$, let $l=\operatorname{lcm}\left(\left(\mathbf{r}_{1}\right)_{v_{1}},\left(\mathbf{r}_{2}\right)_{v_{2}}\right)$ and

$$
l\left(\mathbf{r}_{1}, \mathbf{r}_{2}\right)= \begin{cases}\frac{l}{\left(\mathbf{r}_{1}\right)_{v_{1}}} \cdot\left(\mathbf{r}_{1}\right)_{u} & \text { if } u \in V\left(G_{1}\right), \\ \frac{l}{\left(\mathbf{r}_{2}\right)_{v_{2}}} \cdot\left(\mathbf{r}_{2}\right)_{u} & \text { if } u \in V\left(G_{2}-v_{2}\right) .\end{cases}
$$

Now we are ready to state the main result of this article.

Theorem 2.3. Let $G_{1}, G_{2}$ be two connected graphs and $v_{i}$ a vertex of $G_{i}$. If $G$ is equal to $G_{1} \vee_{v} G_{2}$, then

$$
\mathcal{A}(G)=\left\{\left(\mathbf{a}+{ }_{v} \mathbf{b}, l\left(\mathbf{r}_{1}, \mathbf{r}_{2}\right) \mid \mathbf{a}+{ }_{v} \mathbf{b} \in \mathbb{N}_{+}^{|V(G)|} \text { and }\left(\mathbf{a}, \mathbf{r}_{1}\right) \in \mathcal{A}_{v_{1}}\left(G_{1}\right),\left(\mathbf{b}, \mathbf{r}_{2}\right) \in \mathcal{A}_{v_{2}}\left(G_{2}\right)\right\} .\right.
$$


Proof. Let $\left(\mathbf{d}_{1}, \mathbf{r}_{1}\right)$ and $\left(\mathbf{d}_{2}, \mathbf{r}_{2}\right)$ be $v$-arithmetical structures on $G_{1}$ and $G_{2}$, respectively. We will prove that $\left(\mathbf{d}_{1}+{ }_{v} \mathbf{d}_{2}, l\left(\mathbf{r}_{1}, \mathbf{r}_{2}\right)\right)$ is an arithmetical structure on $G$. First

$$
\begin{aligned}
\operatorname{det}\left(G, \mathbf{d}_{1}+{ }_{v} \mathbf{d}_{2}\right) & =\operatorname{det}\left(G_{1}-v_{1}, \mathbf{d}_{1}^{\prime}\right) \cdot \operatorname{det}\left(G_{2}, \mathbf{d}_{2}\right)+\operatorname{det}\left(G_{2}-v_{2}, \mathbf{d}_{2}^{\prime}\right) \cdot \operatorname{det}\left(G_{1}, \mathbf{d}_{1}\right) \\
& =\operatorname{det}\left(G_{1}-v_{1}, \mathbf{d}_{1}^{\prime}\right) \cdot 0+\operatorname{det}\left(G_{2}-v_{2}, \mathbf{d}_{2}^{\prime}\right) \cdot 0=0
\end{aligned}
$$

where $\mathbf{d}_{i}^{\prime}$ is the vector obtained by erasing the entry $v_{i}$. It is not difficult to see that

$$
L(G, X)=\left(\begin{array}{ccc}
L\left(G_{1}-v_{1}, X\right) & \mathbf{m}_{1}^{t} & \mathbf{0} \\
\mathbf{m}_{1} & x_{v} & \mathbf{m}_{2} \\
\mathbf{0} & \mathbf{m}_{2}^{t} & L\left(G_{2}-v_{2}, X\right)
\end{array}\right)
$$

for some vectors $\mathbf{m}_{1}, \mathbf{m}_{2}$ of integers such that

$$
L\left(G_{1}, X\right)=\left(\begin{array}{cc}
L\left(G_{1}-v_{1}, X\right) & \mathbf{m}_{1}^{t} \\
\mathbf{m}_{1} & x_{v}
\end{array}\right) \text { and } L\left(G_{2}, X\right)=\left(\begin{array}{cc}
x_{v} & \mathbf{m}_{2} \\
\mathbf{m}_{2}^{t} & L\left(G_{2}-v_{2}, X\right)
\end{array}\right) .
$$

Thus

$$
L\left(G, \mathbf{d}_{1}+{ }_{v} \mathbf{d}_{2}\right) l\left(\mathbf{r}_{1}, \mathbf{r}_{2}\right)^{t}=\left(\begin{array}{ccc}
L\left(G_{1}-v_{1}, X\right) & \mathbf{m}_{1}^{t} & \mathbf{0} \\
\mathbf{m}_{1} & x_{v} & \mathbf{m}_{2} \\
\mathbf{0} & \mathbf{m}_{2}^{t} & L\left(G_{2}-v_{2}, X\right)
\end{array}\right) l\left(\mathbf{r}_{1}, \mathbf{r}_{2}\right)^{t}=0 .
$$

Now, for the other containment we have the following arguments. Let $(\mathbf{d}, \mathbf{r})$ be an arithmetical structure on $G$. By [4, Proposition 3.3], $L(G, \mathbf{d})$ is an almost non-singular $M$-matrix, that is, a singular $M$-matrix with all its proper principal minors positive. Let $\mathbf{d}_{i}^{\prime}$ be the vector obtained from $\mathbf{d}$ that only contains the entries in the vertices of $G_{i}-v_{i}$. Since $L\left(G_{1}-v_{1}, \mathbf{d}_{1}^{\prime}\right)$ and $L\left(G_{2}-v_{2}, \mathbf{d}_{2}^{\prime}\right)$ are submatrices of $L(G, \mathbf{d})$, then $\operatorname{det}\left(G_{i}-v_{i}, \mathbf{d}_{i}^{\prime}\right)$ is positive. On the other hand, let $0 \leq t \leq 1$ and

$$
\mathbf{d}_{1}(t)_{u}=\left\{\begin{array}{ll}
\mathbf{d}_{u} & \text { if } u \neq v, \\
t \mathbf{d}_{v} & \text { if } u=v,
\end{array} \quad \text { and } \quad \mathbf{d}_{2}(t)_{u}= \begin{cases}\mathbf{d}_{u} & \text { if } u \neq v, \\
(1-t) \mathbf{d}_{v} & \text { if } u=v,\end{cases}\right.
$$

vectors that depend on $t$ and such that $\mathbf{d}=\mathbf{d}_{1}(t)+{ }_{v} \mathbf{d}_{2}(t)$. By Lemma 2.1

$$
0=\operatorname{det}(G, \mathbf{d})=\operatorname{det}\left(G_{1}-v_{1}, \mathbf{d}_{1}^{\prime}\right) \cdot \operatorname{det}\left(G_{2}, \mathbf{d}_{2}(t)\right)+\operatorname{det}\left(G_{2}-v_{2}, \mathbf{d}_{2}^{\prime}\right) \cdot \operatorname{det}\left(G_{1}, \mathbf{d}_{1}(t)\right)
$$

Thus

$$
\frac{\operatorname{det}\left(G_{1}, \mathbf{d}_{1}(t)\right)}{\operatorname{det}\left(G_{1}-v_{1}, \mathbf{d}_{1}^{\prime}\right)}=-\frac{\operatorname{det}\left(G_{2}, \mathbf{d}_{2}(t)\right)}{\operatorname{det}\left(G_{2}-v_{2}, \mathbf{d}_{2}^{\prime}\right)}
$$

Since $\operatorname{det}\left(G_{i}-v_{i}, \mathbf{d}_{i}^{\prime}\right)$ is positive, then $\operatorname{det}\left(G_{1}, \mathbf{d}_{1}(t)\right) \operatorname{det}\left(G_{2}, \mathbf{d}_{2}(t)\right) \leq 0$. Since $L\left(G_{1}, \mathbf{d}_{1}(1)\right)$ is a submatrix of $L(G, \mathbf{d})$, then $\operatorname{det}\left(G_{1}, \mathbf{d}_{1}(1)\right)>0$. Without loss of generality we can assume that $\operatorname{det}\left(G_{1}, \mathbf{d}_{1}(0)\right) \leq 0$. Since

$$
f(t)=\operatorname{det}\left(G_{1}, \mathbf{d}_{1}(t)\right)=\operatorname{det}\left(G_{1}-v_{1}, \mathbf{d}_{1}^{\prime}\right) t \mathbf{d}_{v}+\operatorname{det}\left(G_{1}, \mathbf{d}_{1}(0)\right)
$$

is a linear function of $t$, then there exists a rational number $0 \leq t^{*}<1$ such that $\operatorname{det}\left(G_{1}, \mathbf{d}_{1}\left(t^{*}\right)\right)=0$, and therefore $\operatorname{det}\left(G_{2}, \mathbf{d}_{2}\left(t^{*}\right)\right)=0$.

Finally, since $L(G, \mathbf{d})$ has rank $|V|-1$, the rest follows because $l\left(\mathbf{r}_{1}, \mathbf{r}_{2}\right)$ is a positive integral vector if and only if $\mathbf{r}_{1}, \mathbf{r}_{2}$ are positive integral vectors.

Since $\mathcal{A}(G) \subseteq \mathcal{A}_{v}(G)$, then by Theorem 2.3

$$
\mathcal{A}\left(G_{1}\right)+{ }_{v} \mathcal{A}\left(G_{2}\right) \subseteq \mathcal{A}(G) .
$$

This relation allows having a good approximation of the arithmetical structures on a connected graph in terms of the arithmetical structures on their 2-connected components. In general, Theorem 2.3 allows decomposing the problem of finding the arithmetical structures on a connected graph into the problem of 
finding the rational arithmetical structures on its 2-connected components. In other words, we transform a problem with a global condition into several local conditions. However, is complex to describe these generalized arithmetical structures.

The next example illustrates that the equality in $\mathcal{A}\left(G_{1}\right)+{ }_{v} \mathcal{A}\left(G_{2}\right) \subseteq \mathcal{A}(G)$ does not necessarily hold.

Example 2.4. Consider the graph $G$ given in Figure Q 2 , obtained by identifying two of the three vertices of a two complete graphs. That is, $G$ has a cut vertex and two complete graphs with three vertices as blocks.

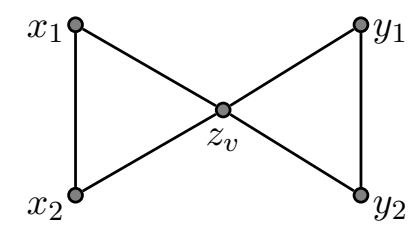

Figure 2. The wedge of two complete graphs with three vertices.

Its generalized Laplacian matrix is given by

$$
L(G, X)=\left(\begin{array}{ccccc}
x_{1} & -1 & -1 & 0 & 0 \\
-1 & x_{2} & -1 & 0 & 0 \\
-1 & -1 & z_{v} & -1 & -1 \\
0 & 0 & -1 & y_{1} & -1 \\
0 & 0 & -1 & -1 & y_{2}
\end{array}\right)
$$

It is not difficult to check that the vectors $\mathbf{d}=(2,3,2,3,7)$ and $\mathbf{r}=(4,3,5,2,1)$ form an arithmetical structure for $G$. However this arithmetical structure is not in $\mathcal{A}\left(K_{3}\right)+{ }_{v} \mathcal{A}\left(K_{3}\right)$. The arithmetical structure given by $(\mathbf{d}, \mathbf{r})$ comes from the rational arithmetical structures on $K_{3}$

$$
((2,3,7 / 5),(4,3,5)) \text { and }(3 / 5,3,7),(5,2,1)) \text {. }
$$

The rational arithmetical structures on a graph are as important as their arithmetical structures. Moreover, any rational arithmetical structure can be extended to an arithmetical structure on some induced supergraph.

Theorem 2.5. Let $G$ be a graph and $U \subseteq V(G)$. If $(\mathbf{d}, \mathbf{r})$ is a $U$-rational arithmetical structure on $G$, then there exists an induced supergraph $H$ of $G$ and an arithmetical structure $\left(\mathbf{d}^{\prime}, \mathbf{r}^{\prime}\right)$ of $H$ such that

$$
\mathbf{d}_{w}=\mathbf{d}_{w}^{\prime}
$$

for all $w \in V(G) \backslash U$.

Proof. Let $u \in U$ and $q=1-\left(\mathbf{d}_{u}-\left\lfloor\mathbf{d}_{u}\right\rfloor\right)$. In 1202 in the book Liber Abaci, Fibonacci discovered an algorithm that shows that every ordinary fraction has an Egyptian Fraction form, see for instance [8] for a proof of its correctness. That is, there exist $m \in \mathbb{N}_{+}$and $\left\{a_{1}, \ldots, a_{m}\right\} \subset \mathbb{N}_{+}$such that

$$
q=\sum_{i=1}^{m} \frac{1}{a_{i}} .
$$

Now, consider the star graph $S_{m}$ with center in $v$ and with $m$ leaves. It is not difficult to see that $\mathbf{f}=\left(1, a_{1}, \ldots, a_{m}\right)$ and $\mathbf{s}=\left(c, \frac{c}{a_{1}}, \ldots, \frac{c}{a_{m}}\right)$, where $c=\operatorname{lcm}\left\{a_{1}, \ldots, a_{m}\right\}$ is a $v$-rational arithmetical 
structure on $S_{m}$.

$$
\left(\begin{array}{cccc}
q & -1 & \cdots & -1 \\
-1 & a_{1} & 0 & 0 \\
\vdots & 0 & \ddots & 0 \\
-1 & 0 & 0 & a_{m}
\end{array}\right)\left(\begin{array}{c}
c \\
\frac{c}{a_{1}} \\
\vdots \\
\frac{c}{a_{m}}
\end{array}\right)=\mathbf{0}
$$

Let $H_{u}=w\left(G, u ; S_{m}, v\right)$. Clearly $H_{u}$ is an induced supergraph of $G$ and by Theorem 2.3, the vectors $\mathbf{d}_{u}^{\prime}=\mathbf{d}+{ }_{v} \mathbf{f}$ and $\mathbf{r}_{u}^{\prime}=l(\mathbf{r}, \mathbf{s})$ form a $(U-u)$-rational arithmetical structure on $H_{u}$. By successive applications of this procedure we can get a supergraph $H$ of $G$ and an arithmetical structure which satisfies the conditions that we want.

We say that the arithmetical structure $\left(\mathbf{d}^{\prime}, \mathbf{r}^{\prime}\right)$ of $H$ is an extension of the $U$-rational arithmetical structure $(\mathbf{d}, \mathbf{r})$ on $G$. In [7, Section 4] there is given a description of the $l$-part of the group of components of a graph $G$ with a cut vertex $v$ in terms of some supergraphs $\tilde{G}_{1}, \ldots, \tilde{G}_{s}$ of the connected components of $G-v$. More precisely, if $G_{1}, \ldots, G_{s}$ are subgraphs of $G$ such that $G=\vee_{v} G_{i}$, then the $\tilde{G}_{1}, \ldots, \tilde{G}_{s}$ are graphs obtained from the $G_{i}$ 's by adding some path that begins in $v$. This corresponds in some sense to an extension (similar to that given in Theorem 2.5, but using paths instead of star graphs) of the $v$-rational arithmetical structures on the 2-connected components of $G$ in order to get an arithmetical structure on the $\tilde{G}_{i}$ 's.

Example 2.6. Consider the rational arithmetical structure (see Figure 3.(a)) of the cycle with four vertices given by $\mathbf{d}=\left(\frac{1}{3}, 6, \frac{5}{3}, 9\right)$ and $\mathbf{r}=(15,3,3,2)$.
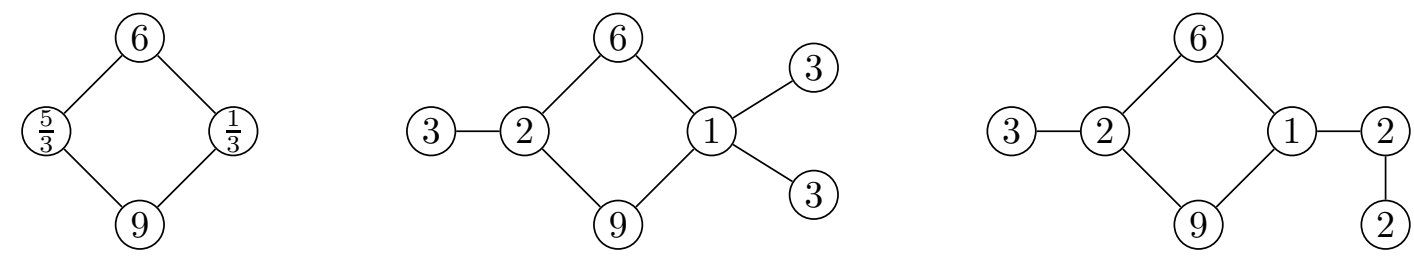

Figure 3. (a) Rational arithmetical structures on $C_{4}$. (b) Supergraph of $C_{4}$. (c) Supergraph of $C_{4}$.

In Figure [3. (b) and 3. (c) we present two extensions of the rational arithmetical structure on $C_{4}$, one using star graphs and the other using paths as a way to construct the induced supergraph. The first one is given by $\mathbf{d}=(3,3,1,6,2,9,3)$ and $\mathbf{r}=(5,5,15,3,3,2,1)$ and the second one is given by $\mathbf{d}=(2,2,1,6,2,9,3)$ and $\mathbf{r}=(5,10,15,3,3,2,1)$.

One of the most natural families of graphs with cut vertices are the trees, therefore in the next section we deal with this particular case.

\section{Arithmetical structures on a tree}

In this section we study a particular case of graphs with connectivity one: the trees. We give a description of the arithmetical structures on a star graph and give a partial description of the arithmetical structures on a general tree in terms of its star graphs.

The simplest tree consists of a simple edge, let us say $P_{2}=u v$. It is not difficult to prove that

$$
\mathcal{A}\left(P_{2}\right)=\{((1,1),(1,1))\} \text {. }
$$


Furthermore, it is not hard to prove that the $\{u\}$-rational arithmetical structures on $P_{2}$ are equal to

$$
\mathcal{A}_{u}\left(P_{2}\right)=\left\{(\mathbf{d}, \mathbf{r}) \in \mathbb{Q}_{+}^{2} \times \mathbb{N}_{+}^{2} \mid \mathbf{d}=\left(\frac{1}{d}, d\right), \mathbf{r}=(d, 1) \text { for some } d \in \mathbb{N}_{+}\right\} .
$$

Let $T$ be any tree with $V$ as its vertex set. Since the blocks of $T$ are its edges, applying Theorem 2.3 we get that the arithmetical structures on $T$ must satisfy the following equations:

Let $\vec{T}$ be the digraph obtained from $T$ by replacing its edges by arcs in both directions and

$$
a: E(\vec{T}) \rightarrow \mathbb{Q}_{+}
$$

be a weigh function on the arcs of $\vec{T}$.

Proposition 3.1. If $T$ is a tree, then

$$
\mathcal{A}(T)=\left\{(\mathbf{d}, \mathbf{r}) \in \mathbb{N}_{+}^{|V(T)|} \times \mathbb{N}_{+}^{|V(T)|} \mid \sum_{u v \in E(T)} \mathbf{a}_{u, v}=\mathbf{d}_{v} \forall v \in V(T) \text { and } \mathbf{a}_{u, v} \mathbf{a}_{v, u}=1 \forall u v \in E(T)\right\} .
$$

Proof. This follows by Theorem 2.3 and the previous description of the arithmetical structures on an edge.

Unfortunately, in general it is not easy to handle this description. Decomposing a tree into some of its star graphs gives us a better way to try to find a description of the arithmetical structures on $T$. The next result give us a description of the arithmetical structures on a star graph.

Corollary 3.2. If $S_{m}$ is the star graph with center at $v$ and $1, \ldots, m$ are its leaves, then

$$
\mathcal{A}\left(S_{m}\right)=\left\{(\mathbf{d}, \mathbf{r}) \in \mathbb{N}_{+}^{m+1} \times \mathbb{N}_{+}^{m+1} \mid \mathbf{d}_{v}=\sum_{i=1}^{m} \frac{1}{\mathbf{d}_{i}}, \mathbf{r}_{v}=\operatorname{lcm}\left\{\mathbf{d}_{i}\right\}_{i=1}^{m} \text { and } \mathbf{r}_{i}=\frac{\mathbf{r}_{v}}{\mathbf{d}_{i}}\right\} .
$$

Proof. (つ) If we have $(\mathbf{d}, \mathbf{r}) \in \mathbb{N}_{+}^{m+1} \times \mathbb{N}_{+}^{m+1}$ such that $\mathbf{d}_{v}=\sum_{i=1}^{m} \frac{1}{\mathbf{d}_{i}}$ and $\mathbf{r}_{i}=\frac{\mathbf{r}_{v}}{\mathbf{d}_{i}}$ where $\mathbf{r}_{v}=\operatorname{lcm}\left\{\mathbf{d}_{i}\right\}_{i=1}^{m}$, then

and therefore $(\mathbf{d}, \mathbf{r}) \in \mathcal{A}\left(S_{m}\right)$.

$$
\left(\begin{array}{cccc}
\mathbf{d}_{v} & -1 & \cdots & -1 \\
-1 & \mathbf{d}_{1} & 0 & 0 \\
\vdots & 0 & \ddots & 0 \\
-1 & 0 & 0 & \mathbf{d}_{m}
\end{array}\right)\left(\begin{array}{c}
\mathbf{r}_{v} \\
\frac{\mathbf{r}_{v}}{\mathbf{d}_{1}} \\
\vdots \\
\frac{\mathbf{r}_{v}}{\mathbf{d}_{m}}
\end{array}\right)=\mathbf{0}
$$

$(\subseteq)$ By Proposition 3.1, since all the vertices of $S_{m}$, except its center, have degree one, $\operatorname{det}\left(L\left(S_{m}, \mathbf{d}\right)\right)=0$ if and only if $\mathbf{d}_{v}=\sum_{i=1}^{m} \frac{1}{\mathbf{d}_{i}}$ for some $\mathbf{d}_{i} \in \mathbb{N}_{+}$. Since $L\left(S_{m}, \mathbf{d}\right)$ is an almost non-singular $M$-matrix, the rest follows by checking that $\mathbf{r}$ is in the kernel of $L\left(S_{m}, \mathbf{d}\right)$.

The next example shows how to use a decomposition of a tree into star graphs in order to get its arithmetical structures.

Example 3.3. Let $T$ be a tree decomposed into a star graph with three leaves $S_{3}$ and a star graph with two leaves $S_{2}$, as in Figure 4.(a). Figures 4 present two arithmetical structures obtained from the decomposition of $T$ into star graphs.

Now, Figure 5 shows how the rational arithmetical structures on the two star graphs of $T$ can be composed. 


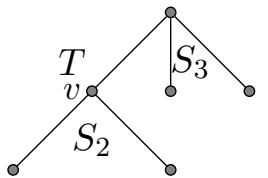

(a) $T$

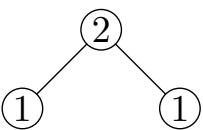

(b) $\mathbf{b}$

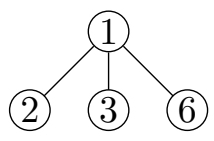

(c) $\mathbf{a}$

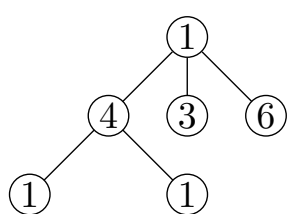

(d) $\mathbf{a}+{ }_{v} \mathbf{b}$

Figure 4. (a) A tree $T$, which can be decomposed into the star graphs $S_{2}$ and $S_{3}$. (b) An arithmetical structure on the star graph $S_{2}$. (c) An arithmetical structure on the star graph $S_{3} .(d)$ The arithmetical structure on $T$ obtained by composing the arithmetical structures on the star graphs $S_{2}$ and $S_{3}$.

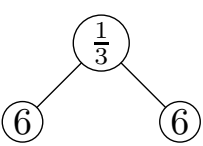

(a) $\mathbf{b}$

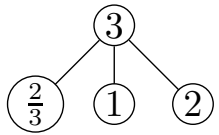

(b) $\mathbf{a}$

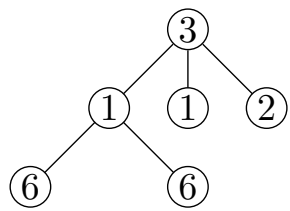

(c) $\mathbf{a}+{ }_{v} \mathbf{b}$

Figure 5. An arithmetical structure on $T$ obtained from two rational arithmetical structures on star graphs.

In the case of trees it is very easy to calculate the critical group associated to any of its arithmetical structures. Lorenzini in [6, Corollary 2.5] proved the next result about the critical group of an arithmetical tree.

Proposition 3.4 (Corollary 2.5). Let $T$ be a (simple) tree. If $(\mathbf{d}, \mathbf{r})$ is an arithmetical structure on $T$, then

$$
|K(T, \mathbf{d})|=\prod_{v \in V(T)} \mathbf{r}_{v}^{\mathbf{d e g}_{v}-2}
$$

where deg is the degree vector of $T$.

\section{REFERENCES}

[1] N. L. Biggs, Algebraic potential theory on graphs. Bull. London Math. Soc. 29 (1997) 641-682.

[2] Benjamin Braun, Hugo Corrales, Scott Corry, Luis David Garca Puente, Darren Glass, Nathan Kaplan, Jeremy L. Martin, Gregg Musiker, and Carlos E. Valencia, Counting arithmetical Structures on Paths and Cycles, preprint. arXiv:1701.06377

[3] H. Corrales and Carlos E. Valencia, On the critical ideals of graphs, Linear Algebra and its Applications 439 (2013) 3870-3892.

[4] H. Corrales and Carlos E. Valencia, Arithmetical structures on graphs, preprint. arXiv:1604.02502.

[5] C. D. Godsil and G. F. Royle, Algebraic Graph Theory, Springer-Verlag, Berlin, 2001.

[6] D. Lorenzini, arithmetical graphs, Math. Ann. 285 (1989), 481-501.

[7] D. Lorenzini, Reduction of points in the group of components of the Néron model of a Jacobian. J. Reine Angew. Math. 527 (2000), 117-150.

[8] J. J. Sylvester, On a point in the theory of vulgar fractions, American Journal of Mathematics 3 (1880), 332-335.

[9] J. Guzmán and C. Klivans, Chip-firing and energy minimization on M-matrices, J. of Combinatorial Theory A 132 (2015) 14-31. 
E-mail address, H. Corrales: hhcorrales@gmail.com

E-mail address, C. Valencial: cvalencia@math.cinvestav.edu.mx, cvalencia75@gmail.com

Departamento de Matemáticas, Centro de Investigación y de Estudios Avanzados del iPn, Apartado Postal 14-740, 07000 Mexico City, D.F.

\footnotetext{
${ }^{1}$ Corresponding author
} 